\title{
Correlating ultrafast calorimetry, viscosity, and structural measurements in liquid GeTe and $\mathrm{Ge}_{15} \mathrm{Te}_{85}$
}

\author{
Hans Weber, ${ }^{1}$ Jiri Orava, ${ }^{1, *}$ Ivan Kaban, ${ }^{1, \dagger}$ Julian Pries, ${ }^{2}$ and A. Lindsay Greer ${ }^{3}$ \\ ${ }^{1}$ IFW Dresden, Institute for Complex Materials, Helmholtzstrasse 20, Dresden 01069, Germany \\ ${ }^{2}$ I. Physikalisches Institut IA, RWTH Aachen, Sommerfeldstrasse 14, 52074 Aachen, Germany \\ ${ }^{3}$ Department of Materials Science and Metallurgy, University of Cambridge, 27 Charles Babbage Road, \\ Cambridge CB3 OFS, United Kingdom
}

(Received 4 June 2018; published 21 September 2018)

\begin{abstract}
Two distinct trends in the temperature dependence of viscosity, measured directly and inferred from calorimetry by analyzing crystallization kinetics, can be correlated with the temperature evolution of the height of the first peak of the $\mathrm{x}$-ray total structure factor for liquid $\mathrm{GeTe}$ and $\mathrm{Ge}_{15} \mathrm{Te}_{85}$. The phase-change chalcogenide GeTe is a high-fragility liquid with the kinetic fragility value of 76 , at the glass-transition temperature, being between those for liquid (Ag,In)-doped $\mathrm{Sb}_{2} \mathrm{Te}$ and $\mathrm{Ge}_{2} \mathrm{Sb}_{2} \mathrm{Te}_{5}$. The viscosity of the high-temperature liquid shows Arrhenius kinetics on cooling to the melting point, and the structure factor conforms to the fragile liquid. For liquid $\mathrm{Ge}_{15} \mathrm{Te}_{85}$, the temperature evolution of the structure factor suggests a transition in the temperature range of about $100 \mathrm{~K}$ above the melting. The crystallization shows a wide range of Arrhenius kinetics in the supercooled liquid region. This finding combined with the dynamic viscosity measurements is interpreted by invoking a weak fragile-to-strong crossover on cooling the liquid $\mathrm{Ge}_{15} \mathrm{Te}_{85}$. The differences in structures and dynamics of liquid GeTe and $\mathrm{Ge}_{15} \mathrm{Te}_{85}$ appear closely correlated to their distinctly different crystallization mechanisms.
\end{abstract}

DOI: 10.1103/PhysRevMaterials.2.093405

\section{INTRODUCTION}

Phase-change memory (PCM), based on chalcogenides such as $\mathrm{Ge}-\mathrm{Sb}$ - $\mathrm{Te}$ and $\mathrm{Ag}-\mathrm{In}-\mathrm{Sb}-\mathrm{Te}$, is a resistive-type memory with Joule-heating-induced reversible glassy-tocrystalline transitions [1]. The phase change (PC) of interest is from the glass to the crystal via supercooled liquid. Change in this direction is the SET (writing) operation in PCM and crystallization, characterized by the crystal growth rate $(U)$, is the rate-limiting step. The RESET operation (erasing) is via melting followed by rapid quenching with the range of critical cooling rates $\sim 10^{8}-10^{12} \mathrm{~K} \mathrm{~s}^{-1}[2]$. Two conflicting requirements must be met in PCM operation: the crystallization time must be short at elevated temperatures, preferably below $\sim 1 \mathrm{~ns}[3-5]$ (typically $<100 \mathrm{~ns}$ ), but the glass must be resistant to spontaneous crystallization at and to some extent above room temperature for data retention.

Chalcogenide $\mathrm{Ge}_{2} \mathrm{Sb}_{2} \mathrm{Te}_{5}$ (GST) is the archetype of the PC supercooled liquid with a high kinetic fragility $(m \approx$ 90) [6], where $m=\left[d\left(\log _{10} \eta\right) / d\left(T_{\mathrm{g}} / T\right)\right]_{T=T_{\mathrm{g}}}$ [7] just above the glass-transition temperature $T_{\mathrm{g}}$, and high growth rate of

\footnotetext{
*Authors to whom correspondence should be addressed: j.orava@ifw-dresden.de

†i.kaban@ifw-dresden.de

Published by the American Physical Society under the terms of the Creative Commons Attribution 4.0 International license. Further distribution of this work must maintain attribution to the author(s) and the published article's title, journal citation, and DOI.
}

$\sim 10^{0} \mathrm{~m} \mathrm{~s}^{-1}$ spanning a remarkably wide temperature range of $(0.60-0.95) T_{\mathrm{m}},\left(T_{\mathrm{m}}=\right.$ melting temperature $)$. The breakdown in the Stokes-Einstein relation [8] can lead to almost $10^{5} \times$ higher $U$ around $T_{\mathrm{g}}$ [6] than would be predicted from the simple coupling relation between the kinetic coefficient of crystal growth $U_{\text {kin }}$ and viscosity $\eta: U_{\text {kin }}(T) \propto \eta(T)^{-1}$ [9].

The focus here is on two compositions from the binary Ge-Te system: GeTe and $\mathrm{Ge}_{15} \mathrm{Te}_{85}$ (at.\%). Liquid GeTe was reported to be a high-fragility liquid with $m \approx 131$ inferred from ultrafast differential scanning calorimetry (FDSC) [10], or $m \approx 104-111$ by molecular-dynamic (MD) simulations [8]. Such a high fragility does not conform well to the relatively lower activation energy for crystal growth $Q_{\mathrm{G}}=$ $171 \mathrm{~kJ} \mathrm{~mol}^{-1}$ [11] reported for GeTe in contrast to the value of $Q_{\mathrm{G}}=227 \mathrm{~kJ} \mathrm{~mol}^{-1}$ for GST [12]. The breakdown in the Stokes-Einstein relation was also suggested by MD simulations in liquid GeTe, where a high atomic mobility is retained even at large supercooling $\left(\Delta T=T_{\mathrm{m}}-T\right)$ unlike what would be expected from the steep increase in viscosity [8]. Recent experimental work did not consider any important role of decoupling for GeTe [10].

The viscosity of supercooled liquid $\mathrm{Ge}_{15} \mathrm{Te}_{85}$ [13] does not fit to single-fragility models, but it can be described by invoking a fragile-to-strong crossover; the liquid is characterized by two distinct values of fragility $m^{\prime}$ (high-temperature end) and $m$ (at $T_{\mathrm{g}}$ ) on either side of the crossover. On cooling, such a liquid progressively changes from being fragile at high temperatures to moderately strong at $T_{\mathrm{g}}$. The values of $m^{\prime} \approx 90$ and $m \approx 50$ were reported for $\mathrm{Ge}_{15} \mathrm{Te}_{85}$ [13], and $m^{\prime} \approx 74$ and $m \approx 37$ for AIST $[14,15]$. 
Although the liquid $\mathrm{Ge}_{15} \mathrm{Te}_{85}$ is not of interest as an active material for PCM because of the sluggish crystallization kinetics, the existence of a crossover similar to that for AIST, in contrast to PC liquid GeTe and $\mathrm{Ge}_{2} \mathrm{Sb}_{2} \mathrm{Te}_{5}$, is of fundamental interest. The existence of a crossover means that fast crystallization can be maintained at high temperatures of the supercooled liquid, and longer incubation times and lower growth rates at larger $\Delta T[2,16]$, due to lower mobility, could help to improve the resistance against spontaneous crystallization. In the presence of a crossover there is no, or negligible, role of the decoupling between $U_{\text {kin }}$ and $\eta$ around $T_{\mathrm{g}}[9,14]$. The shape of $\eta(T)$, especially at large $\Delta T$, seems to underlie the experimentally observed difference in the crystallization mechanisms between two categories of PC chalcogenides [14]: nucleation dominated, represented by GST, and growth dominated, represented by AIST [17].

In this paper, an evaluation of $\eta(T)$ for liquid GeTe and $\mathrm{Ge}_{15} \mathrm{Te}_{85}$ is made by direct measurements of dynamic viscosity combined together with trends inferred from FDSC. The former system conforms to a highly fragile liquid with the experimental fragility being now between those for liquid AIST and GST. The presence of a crossover is confirmed in supercooled liquid $\mathrm{Ge}_{15} \mathrm{Te}_{85}$, revealing a wide range of Arrhenius kinetics at intermediate temperatures and resembling the crossover in liquid AIST. The evaluated liquid fragilities can be correlated with the temperature evolution of the x-ray structure factor.

\section{MATERIALS AND METHODS}

The bulk crystalline $\mathrm{Ge}_{15} \mathrm{Te}_{85}$ master alloy for viscosity and x-ray measurements was prepared from $5 \mathrm{~N}$ purity elements in fused silica ampoules under $\mathrm{Ar}$ atmosphere of $2.5 \times 10^{4} \mathrm{~Pa}$ as described in Ref. [18]. The glass was prepared in a fused silica capillary, with inner diameter of $1.4 \mathrm{~mm}$, with the alloy sealed under Ar atmosphere and then heated up to glowing and quenched in icy water.

Thin-film GeTe, with thickness of $d=5.6 \mu \mathrm{m}$, was deposited by magnetron sputtering using a background pressure of $3 \times 10^{-4} \mathrm{~Pa}$ and an argon pressure of $0.6 \mathrm{~Pa}$.

Calorimetric measurements were made on a Mettler Toledo Flash DSC 1. A sample $(d<10 \mu \mathrm{m}$, a length and a width of up to $50 \times 50 \mu \mathrm{m}^{2}$, and a mass of $<75 \mathrm{ng}$ ) of the glassy $\mathrm{Ge}_{15} \mathrm{Te}_{85}$ and flakes peeled off the thin-film GeTe were used for the calorimetry.

Viscosity measurements of liquid $\mathrm{Ge}_{15} \mathrm{Te}_{85}$ were carried out with an oscillating-cup, described in Ref. [19]. A crucible was heated up to $1223 \mathrm{~K}$ at heating rate of $\Phi=$ $5 \mathrm{~K} \mathrm{~min}^{-1}$ then equilibrated and subsequently measured on cooling at $\Phi=-1 \mathrm{~K} \mathrm{~min}^{-1}$. The experimentally determined temperature-dependent density from Tsuchiya [20] was used for $\eta(T)$ calculations.

$\mathrm{X}$-ray measurements were carried out at the beamline ID11 at the European Synchrotron Radiation Facility (ESRF, Grenoble) with $120-\mathrm{keV}$ energy and in a fused silica capillary, an inner diameter of $1.9 \mathrm{~mm}$, with similar conditions as in Ref. [18]. A commercial hot-gas blower (Cyberstar) heated the sample up to $T=1116 \mathrm{~K}$ followed by cooling to solidification at $658 \mathrm{~K}$, with a dwell time of $3 \mathrm{~min}$ in between the heating and cooling, using a constant heating/cooling rate

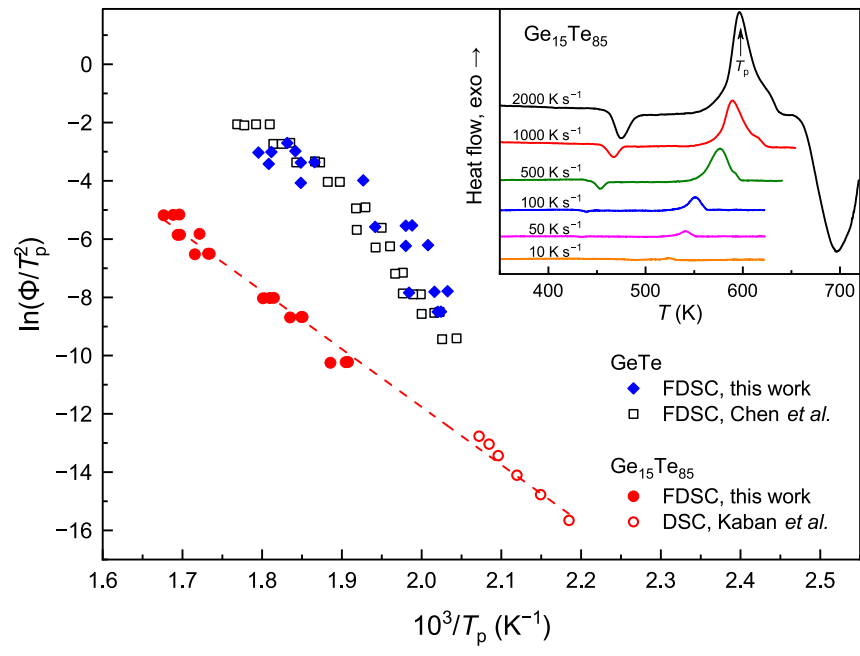

FIG. 1. Kissinger plot of the increasing exothermic peak temperature $T_{\mathrm{p}}$ with increasing heating rate $\Phi$ in DSC. The full symbols, measured by FDSC, are for the present work on $\mathrm{Ge}_{15} \mathrm{Te}_{85}(\Phi=$ $10-2000 \mathrm{~K} \mathrm{~s}^{-1}$, for which the inset shows the corresponding FDSC traces), and on GeTe $\left(\Phi=50-20000 \mathrm{~K} \mathrm{~s}^{-1}\right)$. The data of Chen et al. [10] are from as-deposited single-film GeTe (deposited on $\mathrm{SiO}_{2} / \mathrm{Si}$ by magnetron sputtering, $d=1.4 \mu \mathrm{m}$ ) measured by FDSC $\left(\Phi=20-40000 \mathrm{~K} \mathrm{~s}^{-1}\right)$. The conventional DSC data from melt-spun ribbons of $\mathrm{Ge}_{15} \mathrm{Te}_{85}\left(\Phi=0.033-0.667 \mathrm{~K} \mathrm{~s}^{-1}\right)$ are from Kaban et al. [25] — only the first crystallization peak is considered.

of $\Phi= \pm 5 \mathrm{~K} \mathrm{~min}^{-1}$; the scattered intensities were measured with a FReLoN detector during cooling. Standard data treatment and corrections have been done as in Ref. [18].

\section{RESULTS AND DISCUSSION}

\section{A. Ultrafast calorimetry}

A Kissinger analysis [21] (Fig. 1) of the FDSC traces reveals non-Arrhenius kinetics of the thin-film GeTe crystallization (the traces are not shown) which is similar to that of Chen et al. [10]. Chen et al.'s data are taken for further calculations because of the wider temperature range than the present measurements. The curvature reflects the temperaturedependent $Q_{\mathrm{G}}(T)$, therefore also $\eta(T)$, and it can be obtained by numerical modeling, described in Ref. [6]. The expression of Thompson and Spaepen [22] is used to approximate the driving force for crystallization with the thermodynamic parameters taken from Ref. [23] for GeTe and Ref. [13] for $\mathrm{Ge}_{15} \mathrm{Te}_{85}$.

The inset in Fig. 1 shows FDSC traces of the glassy $\mathrm{Ge}_{15} \mathrm{Te}_{85}$ with the endotherms representing $T_{\mathrm{g}}$ region, the exothermic crystallization peak temperature $T_{\mathrm{p}}$ used for the analysis, and the onset of melting (shown for the highest rate of $\Phi=2000 \mathrm{~K} \mathrm{~s}^{-1}$ only). The low eutectic-melting temperature $T_{\mathrm{e}}=658 \mathrm{~K}$ of $\mathrm{Ge}_{15} \mathrm{Te}_{85}$ is used to evaluate a thermal lag in FDSC which is $\sim 5 \mathrm{~K}$ and less. The crystallization overlaps with the melting at $\Phi>2000 \mathrm{~K} \mathrm{~s}^{-1}$ which makes the estimation of $Q_{\mathrm{G}}(T)$ difficult [24].

The Kissinger analysis of $\mathrm{Ge}_{15} \mathrm{Te}_{85}$ shows Arrhenius kinetics, unlike in GeTe, with a single activation energy of $165 \pm 4 \mathrm{~kJ} \mathrm{~mol}^{-1}$ (shown by the dashed line in Fig. 1-best 


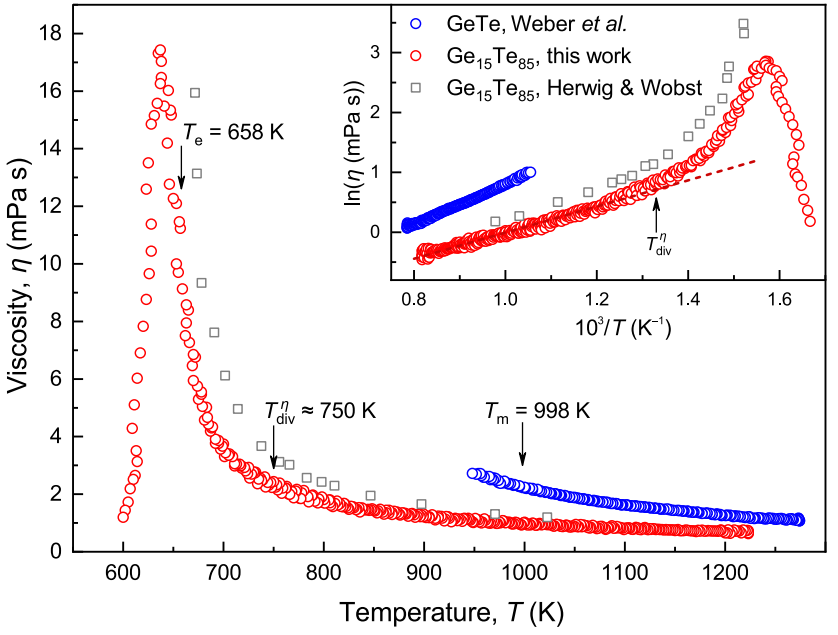

FIG. 2. Temperature-dependent dynamic viscosity $\eta(T)$ for $\mathrm{Ge}_{15} \mathrm{Te}_{85}$ (red open circles), solidifying around $637 \mathrm{~K}$, the data of Herwig and Wobst [28] (grey open squares), and for GeTe [18] (blue open circles) on cooling $\left(\Phi=-1 \mathrm{~K} \mathrm{~min}^{-1}\right)$. The inset shows a logarithmic plot highlighting the temperature, $T_{\mathrm{div}}^{\eta} \approx 750 \mathrm{~K}$, at which $\eta(T)$ diverges from an Arrhenius dependence for $\mathrm{Ge}_{15} \mathrm{Te}_{85}$. The activation energy is $Q_{\eta}=28 \mathrm{~kJ} \mathrm{~mol}^{-1}$ for GeTe (taken over the full $T$ range) and $Q_{\eta}=18 \mathrm{~kJ} \mathrm{~mol}^{-1}$ for $\mathrm{Ge}_{15} \mathrm{Te}_{85}$ at $T>T_{\text {div }}^{\eta}$.

fit to the combined measurements of conventional, only the first crystallization peak is considered, and ultrafast DSC) spanning a wide temperature range of $(0.70-0.91) T_{\mathrm{e}}$. The crystallization shows a complex behavior at the conventional heating rates [25] which cannot be easily modeled using the simple numerical modeling from Refs. [6]. Therefore, only FDSC data are considered for inferring $\eta(T)$. The Arrhenius kinetics also indicates that maximum in crystal growth rate $U_{\max }$ has not been reached yet, which would be reflected by a greater apparent activation energy showing the opposite curvature to $U(T)$ in the Kissinger plot [24]. Orava et al. [14] (inferred from FDSC) and Salinga et al. [26] (optical reflectometry) showed similar Arrhenius kinetics in AIST in the intermediate temperature range of $(0.54-0.60) T_{\mathrm{m}}$ and (0.52-0.68) $T_{\mathrm{m}}$, respectively. Salinga et al. [26] argued that the Arrhenius kinetics cannot be explained by the decoupling, and Orava et al. [14] concluded that such Arrhenius dependence is the consequence of a weak fragile-to-strong crossover in the supercooled liquid.

\section{B. Temperature dependence of viscosity}

The dynamic viscosity $\eta(T)$ of $\mathrm{Ge}_{15} \mathrm{Te}_{85}$ measured in this work (tabulated data are shown in Table S1 of the Supplemental Material [27]) and that of GeTe taken from Weber et al. [18] are shown in Fig. 2. The uncertainty in $\eta(T)$ is below $10 \%$. A supercooling of $\Delta T=21 \mathrm{~K}$ and $50 \mathrm{~K}$ could be achieved for $\mathrm{Ge}_{15} \mathrm{Te}_{85}$ and GeTe, respectively. The sharp decrease in the apparent viscosity below $637 \mathrm{~K}$ for $\mathrm{Ge}_{15} \mathrm{Te}_{85}$ is because the onset of crystallization reduces the viscousflow-related damping in the sample. The crystallization in GeTe is very fast and the apparent viscosity goes virtually to zero within the time resolution of the measurement. The often-quoted data of Herwig and Wobst [28] for $\mathrm{Ge}_{15} \mathrm{Te}_{85}$ are

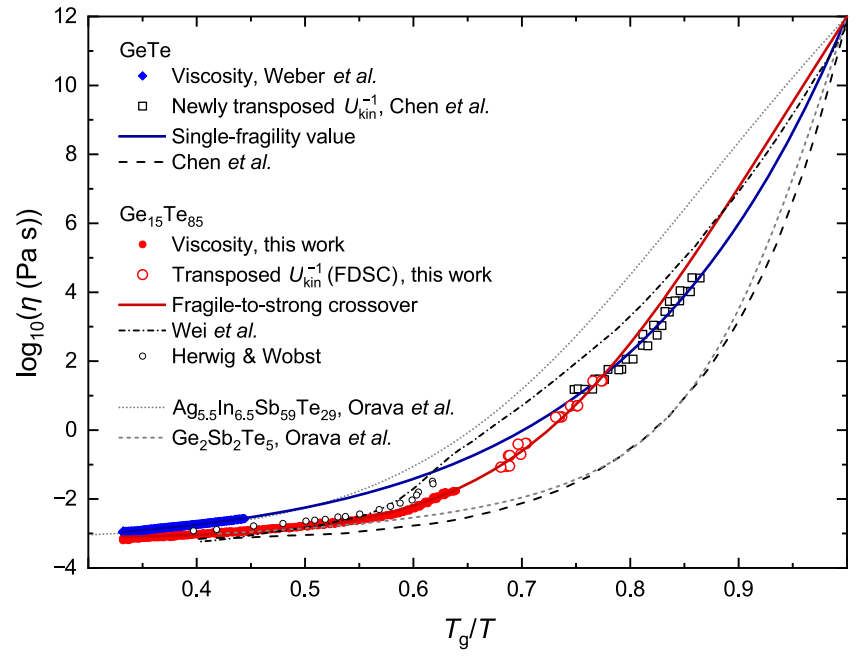

FIG. 3. Angell plot of $\eta(T)$ for liquid chalcogenides. The experimental values of $\eta(T)$ for the single-fragility liquid GeTe $\left(T_{\mathrm{g}}=\right.$ $423 \mathrm{~K}$ ) are shown by the full diamonds-from Weber et al. [18]. The newly transposed $U_{\text {kin }}^{-1}(T)$ (open squares), the data are from Chen et al. [10], was obtained by numerical modeling [6] of the Kissinger plot (Fig. 1). The best fit of $\eta(T)$ to the MYEGA model [29] is shown by the blue solid curve giving $m=76$. The shape of $\eta(T)$ derived by Chen et al. [10] is shown by the dashed curve giving $m=131$. A fragile-to-strong crossover in $G e_{15} T e_{85}\left(T_{\mathrm{g}}=406 \mathrm{~K}\right)$ is represented by the red solid curve (the best fit to the generalized-MYEGA model [32]). The dynamic viscosity $\eta(T)$ (full circles) and the transposed $U_{\text {kin }}^{-1}(T)$ from FDSC (red open circles) are from the present work. A crossover prediction of Wei et al. [13] for $\mathrm{Ge}_{15} \mathrm{Te}_{85}$ is based on the Adam-Gibbs theory and the best fit to calorimetric measurements and viscosity data digitized from Neumann et al. [35]. The data of Herwig and Wobst [28] are shown by the black open circles. The viscosity of $\mathrm{Ge}_{2} \mathrm{Sb}_{2} \mathrm{Te}_{5}$ [6] from Orava et al. and on the crossover in $\mathrm{Ag}_{5.5} \mathrm{In}_{6.5} \mathrm{Sb}_{59} \mathrm{Te}_{29}$ [15] are respectively from FSDC and from the combination of FDSC with $\eta(T)$ measurements.

shown in Fig. 2 for comparison. The present $\eta(T)$ and that of Herwig and Wobst strongly differ below $\approx 710 \mathrm{~K}$ (see the inset plot). The Herwig and Wobst temperature dependence gives a sharper rise in $\eta(T)$ on cooling which is more pronounced on the Angell plot in Fig. 3 (discussed later). With the new high-temperature $\eta(T)$ established, its shape over the entire supercooled liquid region can be described.

For liquid GeTe, $\eta(T)$ can be modeled with a single value of fragility by using the MYEGA (Mauro-Yue-Ellison-GuptaAllan) model [29]: $\log _{10} \eta(\mathrm{T})=\log _{10} \eta_{\infty}+(\mathrm{B} / \mathrm{T}) \exp (\mathrm{C} / \mathrm{T})$, where $\eta_{\infty}$ is the high-temperature limit of viscosity, and $B$ and $C$ are adjustable parameters. The fit is constrained to contain the measured dynamic $\eta(T)$ (full diamonds) and the newly transposed $U_{\text {kin }}(T)^{-1}$ (open squares)- the shape is obtained by numerical modeling [6] from Chen et al. [10]. The best fit to the MYEGA model gives $\log _{10} \eta_{\infty}=-3.317 \pm 0.008($ Pa s $), B=126 \pm 3 \mathrm{~K}$, and $C=$ $1666 \pm 8 \mathrm{~K}$, with $R^{2}=0.9995$ (Fig. 3 ; the blue solid curve). The best fit defines a new value of $m \approx 76$, unlike $m \approx 131$ given by the dashed curve of Chen et al. [10] in Fig. 3. The viscosity suggested by Chen et al. [10] was derived by fitting transposed $U_{\text {kin }}(T)^{-1}$, obtained from FDSC, and high-temperature viscosity inferred from MD simulations by 
Sosso et al. [23] to the MYEGA model. Taking the experimental $U_{\max } \approx 3.3 \mathrm{~m} \mathrm{~s}^{-1}$ at $0.85 T_{\mathrm{m}}$ for as-deposited thinfilm GeTe $(d=30 \mathrm{~nm})$, by Santala et al. [30] using dynamical transmission electron microscopy (TEM), the theoretical fragility can be estimated from the empirical relation presented by Orava and Greer [31] to be $m \approx 50-70$, i.e., between AIST $(m \approx 37)$ and GST $(m \approx 90)$ at $T_{\mathrm{g}}$. Chen et al. [16] suggested the occurrence of a crossover in GeTe nanoparticles, with an average diameter of $10 \mathrm{~nm}$, and they derived $m=78$ by using a generalized-MYEGA model [32] which is surprisingly similar to the present value. It should be noted that viscosity of GST nanoparticles also shows a crossover with $m=57-62$ [33], which is lower than that for the thin film $(m \approx 90)$ [6]. Using a continuous normal crystal growth model [9], assuming no decoupling, the present calculated $U_{\max }$ is $\sim 0.8 \mathrm{~m} \mathrm{~s}^{-1}$ at $0.89 T_{\mathrm{m}}$, which is close to the value reported by Santala et al. [30].

For liquid $G e_{15} T e_{85}$, unlike for $\mathrm{GeTe}$, it was suggested that $\eta(T)$ cannot be described with a single value of $m[13,14]$. For example, the best fit of the measured $\eta(T)$ (from Fig. 2) to the MYEGA model is not good, and gives $m=98$ at $T_{\mathrm{g}}$ (the fit is shown in Fig. S1 of the Supplemental Material [27]), i.e., reiterating the ratio of $m^{\prime} / m \approx 2$ for $\mathrm{Ge}_{15} \mathrm{Te}_{85}$ [13]. A fragile-to-strong crossover suggested by Wei et al. [13] is based on the Adams-Gibbs theory applied to calorimetric data and it is shown by the black dash-dotted line in Fig. 3, the strong end being characterized by $m=50$ at $T_{\mathrm{g}}$. Wei et al.'s fit accounts for the peak in the specific heat capacity around $T_{\mathrm{e}}$, also known for liquid $\mathrm{Te}_{80} \mathrm{Ge}_{20-x} \mathrm{~Pb}_{x}$ [34], giving it its characteristic "double-kink" in $\eta(T)$. This may be further exaggerated because they considered the digitized high-temperature $\eta(T)$ taken from the work of Neumann et al. [35] in which the best fit of the Herwig and Wobst [28] data (black open circles in Fig. 3) to an exponential function is presented. A fragile-to-strong crossover can be described by the generalized-MYEGA model [32]: $\log _{10} \eta(T)=$ $\log _{10} \eta_{\infty}+\left\{T\left[W_{1} \exp \left(-C_{1} / T\right)+W_{2} \exp \left(-C_{2} / T\right)\right]\right\}^{-1}$, where $\eta_{\infty}$ has the same meaning as in the MYEGA equation, and $W_{1}, C_{1}, W_{2}$, and $C_{2}$ are fitting parameters. The fitting constraints are the dynamic $\eta(T)$ measured in this work, the transposed $U_{\mathrm{kin}}(T)^{-1}$ from FDSC, and the kinetic fragility of 50 from Wei et al. [13]. The best fit to the generalized-MYEGA gives $\log _{10} \eta_{\infty}=$ $-3.130 \pm 0.006(\mathrm{~Pa} \mathrm{~s}), W_{1}=(3.1 \pm 0.5) \times 10^{-4} \mathrm{~K}^{-1}, C_{1}=$ $330 \pm 60 \mathrm{~K}, W_{2}=0.56 \pm 0.06 \mathrm{~K}^{-1}$, and $C_{2}=4060 \pm 80 \mathrm{~K}$, with $R^{2}=0.9996$, shown by the red solid curve in Fig. 3 . A wide range of Arrhenius kinetics similar to that for AIST $[14,15,26]$ can be distinguished (Fig. 3). Laser-induced crystallization experiments show that GeTe has the highest and $\mathrm{Ge}_{15} \mathrm{Te}_{85}$ has the lowest crystallization rate in the binary GeTe system [36]. The very sluggish kinetics in $\mathrm{Ge}_{15} \mathrm{Te}_{85}$ arises because of the strong behavior of the supercooled liquid in the range of $T_{\mathrm{g}} / T=0.75-1$, contrary to the fragile liquid GeTe.

\section{Temperature evolution of the $x$-ray structure factor}

The origin of a crossover in liquid chalcogenides remains unclear. It has been suggested that these are transitions between high-temperature metallic and low-temperature semiconducting liquids with directional covalent bonding [14].

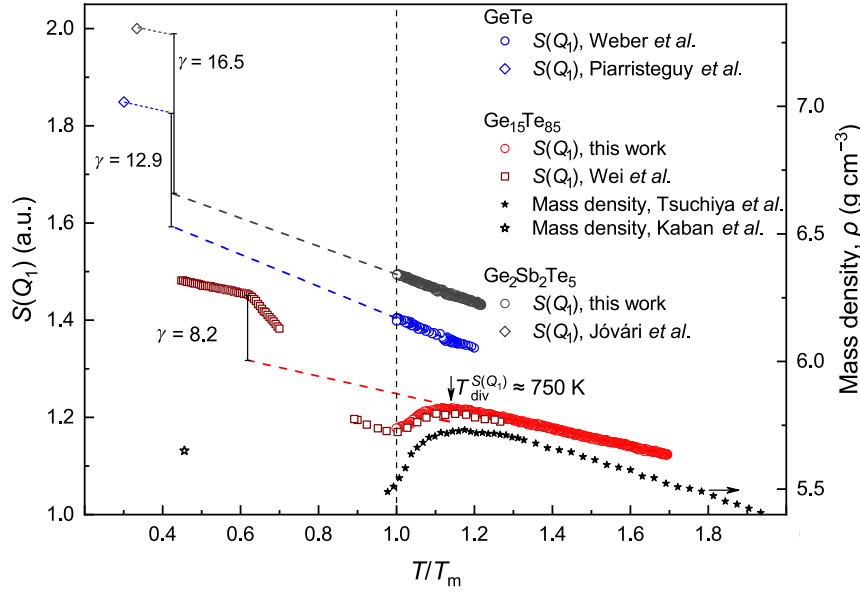

FIG. 4. Left-hand abscissa: Temperature evolution of the height of the first peak $S\left(Q_{1}\right)$ of the x-ray structure factor for GeTe, $\mathrm{Ge}_{15} \mathrm{Te}_{85}$, and $\mathrm{Ge}_{2} \mathrm{Sb}_{2} \mathrm{Te}_{5}$. The data points of $\mathrm{Ge}_{15} \mathrm{Te}_{85}$ are vertically displaced by -0.2 for clarity. The data on the high-temperature liquid GeTe are taken from Weber et al. [18] on cooling $(\Phi=$ $-5 \mathrm{~K} \mathrm{~min}^{-1}$ ) and the room-temperature point is from Piarristeguy et al. [40] (as-deposited thin-film GeTe, $d=1-3 \mu \mathrm{m}$ ); extrapolated to $T_{\mathrm{g}}$. The data on glassy (measured on heating, $\Phi=20 \mathrm{~K} \mathrm{~min}^{-1}$ ) and supercooled (measured on cooling, $\Phi=-20 \mathrm{~K} \mathrm{~min}^{-1}$ ) $\mathrm{Ge}_{15} \mathrm{Te}_{85}$ are from Wei et al. [39] from measurements of bulk samples. The amorphous $\mathrm{Ge}_{2} \mathrm{Sb}_{2} \mathrm{Te}_{5}$ (as-deposited thin film, $d=1-3 \mu \mathrm{m}$ ) is from Jóvári et al. [54], extrapolated to $T_{\mathrm{g}}$ using the temperature coefficient of $\mathrm{Ge}_{15} \mathrm{Te}_{85}$. The structural fragility index $\gamma$ is highlighted by the vertical lines. Linear extrapolations of the high-temperature-liquids trends are shown by the corresponding dashed lines. Right-hand abscissa: Temperature dependence of mass density for liquid [20] and glassy [52] $\mathrm{Ge}_{15} \mathrm{Te}_{85}$.

Mauro et al. [37] demonstrated in several metallic-glassforming liquids that the temperature evolution of the total structure factor $S(Q)$ can be correlated with kinetic fragility. A structural fragility index has been defined from the height of the first peak $S\left(Q_{1}\right)$ of the total structure factor as $\gamma=\left[\left(S\left(Q_{1}\right)_{\text {glass }}-S\left(Q_{1}\right)_{\text {extrapolated liquid }}\right) / S\left(Q_{1}\right)_{\text {glass }}\right] 100$ at $T_{\mathrm{g}}$ [37]; more details can be found in Refs. [37,38]. Though the chalcogenide semiconducting liquids and glasses are characterized by a short-range order (SRO) essentially different from that of liquid and glassy metals, the concept of the structural fragility seems to also provide a useful insight into structural evolution of liquid and glassy chalcogenides with temperature.

The temperature evolutions of the height of the first peak $S\left(Q_{1}\right)$ for $\mathrm{Ge}_{15} \mathrm{Te}_{85}$, GeTe (taken from Ref. [18]) and $\mathrm{Ge}_{2} \mathrm{Sb}_{2} \mathrm{Te}_{5}$ are shown in Fig. 4. A three-dimensional view of the total x-ray structure factor for $\mathrm{Ge}_{15} \mathrm{Te}_{85}$ on cooling between 1116 and $657 \mathrm{~K}$ is shown in the Supplemental Material (Fig. S2) [27]. The $S\left(Q_{1}\right)$ for $\mathrm{Ge}_{15} \mathrm{Te}_{85}$ glass and the low-temperature liquid from Wei et al. [39] are also shown. We are not aware of any data for the temperature dependence of the $S(Q)$ for GeTe glass. Therefore $S\left(Q_{1}\right)$ of amorphous thin-film GeTe at room temperature reported by Piarristeguy et al. [40] and its extrapolation to $T_{\mathrm{g}}$ using the temperature coefficient of $\mathrm{Ge}_{15} \mathrm{Te}_{85}$ glass are plotted in Fig. 4. 
For GeTe, $S\left(Q_{1}\right)-T$ follows linear dependence, above $T_{\mathrm{m}}$, and $\gamma=12.9$ (Fig. 4); the large discrepancy between the amorphous phase and the extrapolated liquid indicates a fragile liquid, which is in accordance with the viscosity (Fig. 3). A greater discrepancy between the extrapolations, $\gamma=16.5$, can be seen for the phase-change chalcogenide $\mathrm{Ge}_{2} \mathrm{Sb}_{2} \mathrm{Te}_{5}$ conforming to $m_{\mathrm{Ge}_{2} \mathrm{Sb}_{2} \mathrm{Te}_{5}}=90$ [6] vs $m_{\mathrm{GeTe}}=$ 76. The liquid $\mathrm{Ge}_{2} \mathrm{Sb}_{2} \mathrm{Te}_{5}$ was measured at the beamline ID11 at ESFR, Grenoble, using similar conditions as in Ref. [18].

For $G e_{15} T e_{85}, S\left(Q_{1}\right)-T$ shows a remarkable deviation from the high-temperature linear dependence on cooling. The structural fragility of the extrapolated high-temperature liquid (the red dashed line in Fig. 4) is $\gamma=8.2$. Taking the data of Wei et al. [39] only gives $\gamma=10.2$, demonstrating that the eutectic composition is an apparently stronger liquid relative to GeTe. The divergence temperature $T_{\operatorname{div}}^{S\left(Q_{1}\right)} \approx$ $750 \mathrm{~K}$ (Fig. 4) corresponds to the value obtained by viscosity measurement $T_{\text {div }}^{\eta} \approx 750 \mathrm{~K}$ (see the inset in Fig. 2). This temperature lies in the temperature range of $\sim 600-800 \mathrm{~K}$ in which the anomalous behavior of several physical properties of liquid $\mathrm{Ge}_{15} \mathrm{Te}_{85}$ (e.g., molar volume, compressibility, sound velocity, specific heat capacity) was observed by Tsuchiya et al. [20,41-43]. Characterization of the $S\left(Q_{1}\right)$ evolution for the supercooled liquid region is hindered by the onset of crystallization around $592 \mathrm{~K}\left(0.90 T_{\mathrm{m}}\right)$ on cooling [39]. The Kissinger analysis shows a wide range of Arrhenius kinetics at $(0.70-0.91) T_{\mathrm{m}}$ (Fig. 1). This range coincides with the "no-man's land" in the intermediate temperature range of the $S\left(Q_{1}\right)$ plot (Fig. 4), which could suggest a (nearly) Arrhenius trend (note the different activation energies).

As discussed by Angell and co-workers [13,39] there is a link between thermodynamic properties (represented, e.g., by an excess entropy), structure, and therefore $\eta(T)$ for $\mathrm{Ge}_{15} \mathrm{Te}_{85}$, and other liquids. Structural studies of $\mathrm{Ge}_{15} \mathrm{Te}_{85}$, e.g., Refs. [44-50], show that there is a transition from high-temperature to low-temperature liquid in the temperature range of about $100 \mathrm{~K}$ above $T_{\mathrm{m}}$. Analysis of the partial atomic distributions in liquid $\mathrm{Ge}_{15} \mathrm{Te}_{85}$ [49] reveals developing tetrahedral local order in the low-temperature liquid, similar to that in the glassy state [51]. In particular, the first- and the second-coordination shells for Ge-Te and Te-Te correlations become very pronounced (distinctly separated) on cooling to the melting temperature. Wei et al. [39] analyzed the temperature dependencies of the total structure factors and pair distribution functions for $\mathrm{Ge}_{15} \mathrm{Te}_{85}$, particularly the ratio of the peak heights $S\left(Q_{1}\right) / S\left(Q_{2}\right)$ and the $S\left(Q_{1}\right)$ prepeak, and they also concluded that the crossover in liquid $\mathrm{Ge}_{15} \mathrm{Te}_{85}$ is related to the structural changes on SRO and on the medium-range $(\sim 8 \AA)$ order $(\mathrm{MRO})$ length scale.

A gradual structural change from the high-temperature to the low-temperature liquid $\mathrm{Ge}_{15} \mathrm{Te}_{85}$ between $\approx 750 \mathrm{~K}$ and $T_{\mathrm{m}}$ is seen in the temperature dependencies of $S\left(Q_{1}\right)$ in Fig. 4. Unfortunately, there are no data for the supercooled liquid between 461 and $592 \mathrm{~K}$ because of the occurrence of crystallization at the applied heating/cooling rates in the diffraction experiment [39]. Still, it is reasonable to suppose that after formation of the low-temperature liquid with predominant tetrahedral order [49] around the eutectic temperature, this structure remains until freezing into the glass. This scenario is supported by the temperature dependency of the density of liquid [20] and glassy $\mathrm{Ge}_{15} \mathrm{Te}_{85}$ [52] presented in Fig. 4. The temperature evolution of $S\left(Q_{2}\right)$ (Fig. S3 of the Supplemental Material [27]), similar to that in Ref. [49] (see Fig. 3 in the reference), does not reflect so well the "anomalous" structural changes in liquid $\mathrm{Ge}_{15} \mathrm{Te}_{85}$ revealed by $S\left(Q_{1}\right)-T$.

For comparison we note that Georgarakis et al. [53] measured temperature-dependent $S\left(Q_{1}\right)$ in the entire supercooled region of $\mathrm{Zr}_{60} \mathrm{Cu}_{30} \mathrm{Al}_{10}$ with clear $\mathrm{SRO}$ and $\mathrm{MRO}$ evolution on cooling $\left(\Phi \approx-100 \mathrm{~K} \mathrm{~s}^{-1}\right)$. Two structural fragility regimes, a fragile and a strong end, could be distinguished between the high- (above $T_{\mathrm{m}}$ ) and the low-temperature (at large $\Delta T$ ) liquid, respectively. The temperature of an "interplay" between the two $\gamma$ ends, estimated by linear extrapolations, correlates with the temperature of a sharp increase in $\eta(T)$ hinting at a possible crossover in the liquid and demonstrating the correlation between $S\left(Q_{1}\right)$ and $\eta(T)$.

\section{CONCLUSIONS}

The present work shows that the trends in the temperaturedependent viscosity, measured directly and inferred from calorimetry, can be correlated with the temperature evolution of the structure factor in the liquid chalcogenides. Two distinct temperature dependent behaviors of viscosity and structure factor are found between liquid $\mathrm{GeTe}$ and $\mathrm{Ge}_{15} \mathrm{Te}_{85}$. The chalcogenide GeTe conforms to a high-fragility liquid ( $m=$ 76) at $T_{\mathrm{g}}$, and the high-temperature liquid, above $T_{\mathrm{m}}$, shows Arrhenius kinetics in $\eta(T)$ and linear temperature evolution of $S\left(Q_{1}\right)$. For $\mathrm{Ge}_{15} \mathrm{Te}_{85}$, the temperature evolution of $S\left(Q_{1}\right)$ suggests a structural change from high-temperature metallic to low-temperature semiconducting liquid on cooling. The crystallization shows Arrhenius kinetics in the intermediate temperature range of the supercooled region because of the presence of the weak fragile-to-strong crossover on cooling. A tight correlation between the dynamic and the structural properties of the liquids studied explains the distinct crystallization mechanisms - the presence of a crossover and slow crystallization for $\mathrm{Ge}_{15} \mathrm{Te}_{85}$, and the absence of a crossover and fast crystallization for GeTe, which is important for phasechange memory applications and its performance.

\section{ACKNOWLEDGMENTS}

I.K. acknowledges the financial support by the German Research Foundation DFG (Contract No. Ka-3209/6-1). Professor M. Wuttig (RWTH Aachen) is greatly acknowledged for discussion. High-temperature X-ray diffraction measurements were done on the beamline ID11 at the European Synchrotron Radiation Facility (ESRF), Grenoble, France. We are grateful to Dr. J. Wright for the assistance with using the beamline and data treatment. We thank B. Opitz, B. Bartusch, and C. G. F. Blum for technical support. Dr. $\mathrm{S}$. Wei is acknowledged for providing the XRD data for $\mathrm{Ge}_{15} \mathrm{Te}_{85}$ published in Ref. [39]. 
[1] M. Wuttig and N. Yamada, Nat. Mater. 6, 824 (2007).

[2] J. Orava and A. L. Greer, Acta Mater. 139, 226 (2017).

[3] D. Loke, T. H. Lee, W. J. Wang, L. P. Shi, R. Zhao, Y. C. Yeo, T. C. Chong, and S. R. Elliott, Science 336, 1566 (2012).

[4] G. Bruns, P. Merkelbach, C. Schlockermann, M. Salinga, M. Wuttig, T. D. Happ, J. B. Philipp, and M. Kund, Appl. Phys. Lett. 95, 043108 (2009).

[5] F. Rao, K. Ding, Y. Zhou, Y. Zheng, M. Xia, S. Lv, Z. Song, S. Feng, I. Ronneberger, R. Mazzarello, W. Zhang, and E. Ma, Science 358, 1423 (2017).

[6] J. Orava, A. L. Greer, B. Gholipour, D. W. Hewak, and C. E. Smith, Nat. Mater. 11, 279 (2012).

[7] C. A. Angell, Science 267, 1924 (1995).

[8] G. C. Sosso, J. Behler, and M. Bernasconi, Phys. Status Solidi B 249, 1880 (2012).

[9] M. D. Ediger, P. Harrowell, and L. Yu, J. Chem. Phys. 128, 034709 (2008).

[10] Y. Chen, G. Wang, L. Song, X. Shen, J. Wang, J. Huo, R. Wang, T. Xu, S. Dai, and Q. Nie, Cryst. Growth Des. 17, 3687 (2017).

[11] Q. M. Lu and M. Libera, J. Appl. Phys. 77, 517 (1995).

[12] J. Kalb, F. Spaepen, and M. Wuttig, Appl. Phys. Lett. 84, 5240 (2004).

[13] S. Wei, P. Lucas, and C. A. Angell, J. Appl. Phys. 118, 034903 (2015).

[14] J. Orava, D. W. Hewak, and A. L. Greer, Adv. Funct. Mater. 25, 4851 (2015).

[15] J. Orava, H. Weber, I. Kaban, and A. L. Greer, J. Chem. Phys. 144, 194503 (2016).

[16] B. Chen, D. de Wal, G. H. ten Brink, G. Palasantzas, and B. J. Kooi, Cryst. Growth Des. 18, 1041 (2018).

[17] G.-F. Zhou, Mater. Sci. Eng. A 304-306, 73 (2001).

[18] H. Weber, M. Schumacher, P. Jóvári, Y. Tsuchiya, W. Skrotzki, R. Mazzarello, and I. Kaban, Phys. Rev. B 96, 054204 (2017).

[19] S. Gruner, J. Alloy Compds. 480, 629 (2009).

[20] Y. Tsuchiya, J. Non-Cryst. Solids 312-314, 212 (2002).

[21] J. Orava and A. L. Greer, in Handbook of Thermal Analysis and Calorimetry, edited by S. Vyazovkin, N. Koba, and C. Schick (Elsevier, Amsterdam, 2018), Chap. 17, p. 685.

[22] C. V. Thompson and F. Spaepen, Acta Metall. 27, 1855 (1979).

[23] G. C. Sosso, G. Miceli, S. Caravati, F. Giberti, J. Behler, and M. Bernasconi, J. Phys. Chem. Lett. 4, 4241 (2013).

[24] J. Orava and A. L. Greer, Thermochim. Acta 603, 63 (2015).

[25] I. Kaban, E. Dost, and W. Hoyer, J. Alloy Compds. 379, 166 (2004).

[26] M. Salinga, E. Carria, A. Kaldenbach, M. Bornöfft, J. Benke, J. Mayer, and M. Wuttig, Nat. Commun. 4, 2371 (2013).

[27] See Supplemental Material at http://link.aps.org/supplemental/ 10.1103/PhysRevMaterials.2.093405 for the dynamic viscosity (Table S1), the single-value fragility best fit (Fig. S1), the temperature evolution of the total structure factor for $\mathrm{Ge}_{15} \mathrm{Te}_{85}$ (Fig. S2), and the temperature dependence of $S\left(Q_{2}\right)$ (Fig. S3).
[28] F. Herwig and M. Wobst, Z. Metallkde. 82, 917 (1991).

[29] J. C. Mauro, Y. Yue, A. J. Ellison, P. K. Gupta, and D. C. Allan, Proc. Natl. Acad. Sci. USA 106, 19780 (2009).

[30] M. K. Santala, B. W. Reed, S. Raoux, T. Topuria, T. LaGrange, and G. H. Campbell, Appl. Phys. Lett. 102, 174105 (2013).

[31] J. Orava and A. L. Greer, J. Chem. Phys. 140, 214504 (2014).

[32] C. Zhang, L. Hu, Y. Yue, and J. C. Mauro, J. Chem. Phys. 133, 014508 (2010).

[33] B. Chen, G. H. ten Brink, G. Palasantzas, and B. J. Kooi, J. Phys. Chem. C 121, 8569 (2017).

[34] L. Battezzati and A. L. Greer, J. Mater. Res. 3, 570 (1988).

[35] H. Neumann, F. Herwig, and W. Hoyer, J. Non-Cryst. Solids 205-207, 438 (1996).

[36] M. Chen, K. A. Rubin, and R. W. Barton, Appl. Phys. Lett. 49, 502 (1986).

[37] N. A. Mauro, M. Blodgett, M. L. Johnson, A. J. Vogt, and K. F. Kelton, Nat. Commun. 5, 4616 (2014).

[38] K. F. Kelton, J. Phys.: Condens. Matter 29, 023002 (2017).

[39] S. Wei, M. Stolpe, O. Gross, W. Hembree, S. Hechler, J. Bednarcik, R. Busch, and P. Lucas, Acta Mater. 129, 259 (2017).

[40] A. A. Piarristeguy, M. Micoulaut, R. Escalier, P. Jóvári, I. Kaban, J. van Eijk, J. Luckas, S. Ravindren, P. Boolchand, and A. Pradel, J. Chem. Phys. 143, 074502 (2015).

[41] Y. Tsuchiya, J. Phys. Soc. Jpn. 60, 227 (1991).

[42] Y. Tsuchiya and H. Saitoh, J. Phys. Soc. Jpn. 62, 1272 (1993).

[43] Y. Tsuchiya, K. Saitoh, and F. Kakinuma, Monatshefte für Chem. 136, 1963 (2005).

[44] H. Neumann, W. Hoyer, W. Matz, and M. Wobst, J. Non-Cryst. Solids 97-98, 1251 (1987).

[45] Y. Kameda, O. Uemura, and T. Usuki, Mater. Trans., JIM 37, 1655 (1996).

[46] S. Yoshioka, Y. Kawakita, M. Kanehira, and S. Takeda, Jpn. J. Appl. Phys. Suppl. 38, 468 (1999).

[47] C. Bichara, J. P. Gaspard, and J. R. Raty, J. Non-Cryst. Solids 312-314, 341 (2002).

[48] C. Bergman, C. Bichara, J. P. Gaspard, and Y. Tsuchiya, Phys. Rev. B 67, 104202 (2003).

[49] I. Kaban, P. Jóvári, W. Hoyer, R. G. Delaplane, and A. Wannberg, J. Phys.: Condens. Matter 18, 2749 (2006).

[50] I. Kaban, W. Hoyer, A. Il'inskii, A. Shpak, and P. Jóvári, J. NonCryst. Solids 353, 1808 (2007).

[51] P. Jóvári, I. Kaban, W. Hoyer, R. G. Delaplane, and A. Wannberg, J. Phys.: Condens. Matter 17, 1529 (2005).

[52] I. Kaban, P. Jóvári, W. Hoyer, and E. Welter, J. Non-Cryst. Solids 353, 2474 (2007).

[53] K. Georgarakis, L. Hennet, G. A. Evangelakis, J. Antonowicz, B. G. Bokas, V. Honkimaki, A. Bytchkov, M. W. Chen, and A. R. Yavari, Acta Mater. 87, 174 (2015).

[54] P. Jóvári, I. Kaban, J. Steiner, B. Beuneu, A. Schöps, and A. Webb, J. Phys.: Condens. Matter 19, 335212 (2007). 